\title{
GRUSSWORT II
}

Am 14. Februar 1887 erschien in der Zeitschrift "Le Temps” ein "Protest der Künstler":

Wir leidenschaftlichen Liebhaber der bisher unangetasteten Schönheit von Paris protestieren mit aller Kraft gegen die Errichtung des unnötigen und ungeheuerlichen Eiffelturms. Wird die Stadt Paris sich wirklich den überspannten, den geschäftstüchtigen Phantastereien einer Maschinenkonstruktion anschließen, um sich für immer zu schänden und zu entehren? Um zu begreifen, was wir kommen sehen, muss man sich für einen Augenblick einen schwindelerregenden, lächerlichen Turm vorstellen, der wie ein riesiger, düsterer Fabrikschlot Paris überragt, muss sich vorstellen, wie alle unsere Monumente gedemütigt, alle unsere Bauten verkleinert werden, bis sie in diesem Albtraum verschwinden.

Wenn es also damals schon das Welterbe-Programm der UNESCO gegeben hätte ...!? Wir müssen aber gar nicht so weit in der Zeit zurückgehen. Schon ein kürzerer Zeitsprung nach 1972 zeigt den immensen Erfolg des Welterbe-Programms der UNESCO: Wer hätte damals gedacht, was aus dieser Initiative 40 Jahre später geworden ist?

Unter den ersten Stätten, die 1978 zum ersten Mal in die Liste des Weltkultur- und -naturerbes der UNESCO aufgenommen wurden, war auch eine deutsche: der Aachener Dom. Das Nominierungsdossier zu dieser ersten deutschen Welterbestätte umfasst gerade einmal sechs, nur teilweise beschriftete Schreibmaschinenseiten. Was waren das noch für Zeiten, als ein Nominierungsdossier gerade einmal sechs halb beschriebene Seiten umfasste!

1978 gab es insgesamt zwölf Kultur- und Naturdenkmäler weltweit, u.a. die Galapagos Inseln, den Yellowstone Nationalpark und weitere drei Natur- und sieben Kulturerbestätten (u.a. die Altstadt von Krakau und die Insel Gorée im Senegal), heute sind es fast 1.000. Und ein Nominierungsdossier umfasst heute gerne einmal 1.000 oder auch 2.000 Seiten! Es ist also einiges geschehen in den bald 40 Jahren seither! Heute sind 981 Kultur- und Naturerbestätten aus 160 Staaten aller Kontinente auf der berühmten UNESCO-Liste verzeichnet. Wichtiger noch: 191 Staaten haben inzwischen das "Internationale Übereinkommen zum Schutz des Kultur- und Naturerbes der Welt“ ("Convention for the Protection of the World Cultural and Natural Heritage“) der UNESCO von 1972 unterzeichnet. 
Das Welterbe-Programm ist eine Erfolgsgeschichte der friedlichen Zusammenarbeit der Staaten dieser Welt ohnegleichen - in einem der sensibelsten und für Ausund Abgrenzungen und auch für gewalttätige Konflikte anfälligsten Bereiche - dem der Kulturpolitik. Ein erster Ansatz von Weltkulturpolitik also! Und dank der Naturerbestätten auch eine „Welt-Naturschutz-Politik“.

In der ganzen Welt ist Kultur noch immer auch ein Vehikel nationaler Selbstbehauptung. Kultur hat oft eine chauvinistische Dimension und diente oft eher der Abgrenzung und Überbietung als der Öffnung und dem Dialog. Seit ihrem Inkrafttreten 1976 trägt die Welterbe-Konvention dazu bei, Kultur aus dem Kontext der nationalen Chauvinismen herauszulösen. Sie trennt den Begriff der Kultur von dem der Nation. Das ist angesichts der Probleme, mit denen wir zur Zeit weltweit konfrontiert sind - denken wir an Syrien, an Mali, an Afghanistan -, nicht hoch genug zu schätzen. Und der Erfolg hält an: Mehr als 60 Prozent der medialen Aufmerksamkeit in Deutschland zum Stichpunkt UNESCO entfällt auf das Welterbe-Programm. Es ist erfolgreich, populär und sichtbar, gibt aber immer wieder auch Anlass für kontroverse Debatten und Konflikte. Dresden und Köln sind nur die bekanntesten Beispiele.

Das Welterbe liegt zunehmend - neben der Wahrnehmung durch Denkmalschützer und Kulturpolitiker - auch im Fokus der Aufmerksamkeit von Parlamentariern und Politikern unterschiedlicher Ressorts. Allein sieben Bundesministerien befassen sich mit dem Welterbe-Programm (AA, BMU, BKM, BMVBS, BMZ, BMBF, BMW), hinzu kommen die Landesressorts, die Kultusministerkonferenz, die kommunale Ebene, Stadtund Landschaftsplaner, Naturschützer, Bildungs- und Forschungsinitiativen, Universitäten und Schulen, Unternehmer und Touristiker. Auch dank der Nominierung deutscher Naturerbestätten ist das Welterbe nicht mehr alleinige kulturpolitische oder Denkmalschutzdomäne. Das Welterbe-Programm der UNESCO ist in der Mitte der Gesellschaft angekommen! Und dies nicht nur in Deutschland! Weltweit warten derzeit noch einmal 1623 Stätten aus 171 Ländern auf ihre Nominierung! Auch in Deutschland wird derzeit die Tentativliste aktualisiert. Zahlreiche Nominierungswünsche werden teils auf lokaler, teils nationaler Ebene geäußert und debattiert.

Warum also wollen alle auf diese berühmte Liste? Der erste und wichtigste Grund liegt sicherlich im Prestigegewinn, den die Adelung durch den Welterbtitel bringt. Es geht eine gewisse Magie von diesem Titel aus (verschwiegen sei nicht: auch Eitelkeiten lassen sich gerne mit dem UNESCO-Titel umschmeicheln).

Prof. Dr. Bernd Freiherr von Droste zu Hülshoff, Gründungsdirektor des Welterbezentrums in Paris, erinnert an die sagenhaften Sieben Weltwunder der Antike, deren magische Wirkung auf die Zeitgenossen und die Nachwelt bis heute anhält: „Herausragende Zeugnisse der Vergangenheit haben oft ein bewegtes Leben. Die ihnen entgegengebrachte Wertschätzung macht sie begehrt." 
Aber, fährt er fort:

So geraten sie immer wieder zwischen die Fronten von exzessiver Nutzung auf der einen und Schutz und Erhaltung auf der anderen Seite. Ihr Schicksal aber darf uns nicht gleichgültig sein [...] Von den sieben Weltwundern der Antike ist heute nur mehr (ein einziges) das Älteste zu bestaunen $[\ldots]$,

nämlich die Pyramiden von Giseh. Verschollen, umgestürzt, abgetragen oder eingeäschert sind alle anderen Weltwunder.

Dem unbestrittenen Prestigegewinn stehen daher auch zahlreiche Aufgaben und Pflichten gegenüber. Ich spreche daher gerne von den "drei Säulen“ des Welterbe-Programms der UNESCO:

1. Schutz (konservatorischer Denkmalschutz, beziehungsweise Naturschutz);

2. Tourismus als nachhaltiger Tourismus (Zugang und Nutzung);

Welterbe-Bildung: Welterbestätten als Orte des interkulturellen Lernens und der interkulturellen Begegnung. Diese dritte Säule des UNESCO-Welterbe-Programms, die Welterbe-Bildung, wird angesichts der Konflikte in dieser Welt immer wichtiger. Durch sie soll der Blick auf die Zukunft gerichtet werden!

Betrachten wir die einzelnen Säulen:

\section{Säule: Schutz}

Dieses Programm war Folge einer konkreten internationalen Hilfsaktion: Als nämlich die Tempelanlagen von Abu Simbel in Nubien durch den geplanten Assuan-Staudamm von Überflutung bedroht waren, rief der Generaldirektor der UNESCO am 8. März 1960 zu einer einmaligen Unterstützungsaktion auf. Fünfzig Länder beteiligten sich mit 80 Millionen US-Dollar an den Rettungsaktionen, darunter auch die Bundesrepublik Deutschland und die DDR, und die riesigen Monumente konnten in einer aufwändigen Aktion zerlegt und an höherer Stelle wieder aufgebaut werden.

Am Anfang stand also eine beispiellose Solidaritätsaktion. Daraus resultierte ein manchmal schmerzhafter, aber letztlich friedensstiftender - partieller Souveränitätsverzicht: Herausragende Kultur- und Naturstätten dieser Erde sind Schätze, so kostbar und wichtig für das Menschheitsgedächtnis, dass sie nicht allein dem Staat oder der Nation gehören, auf deren Territorium sie sich befinden oder denen sie aufgrund ihrer Geschichte zugefallen sind. Die herausragenden Kulturstätten und die großartigen Naturlandschaften dieser Erde sind ideeller Besitz der gesamten Menschheit, der gegenwärtigen und der künftigen Generationen.

Die Veranstaltung hier an der Universität Konstanz soll sensibilisieren für den Schutz und die Bewahrung herausragender Kultur- und Naturgüter, nicht nur die auf der berühmten UNESCO-Liste des Weltkultur und Naturerbes. Die Verantwortung für ihren Schutz fällt unter die Obhut der gesamten Menschheit. Hier liegt der eigentliche Kern der UNESCO-Welterbe-Konvention: Je bedeutender ein Gut ist, umso universeller 
ist seine Gültigkeit. Auf der Grundlage eines universellen Erbebegriffes sind die kooperierenden Staaten bereit, das Eigene in eine Reihe zu stellen mit dem Fremden und diesem die Anerkennung zukommen zu lassen, aus der sich letztlich auch der Stolz auf das Eigene nährt.

\section{Säule: Nachhaltiger Tourismus}

Der Kulturbegriff der Welterbe-Konvention bezieht sich auf kulturelle Vielfalt und kulturellen Pluralismus. Die Liste des Welterbes ist daher auch eine faszinierende Landkarte der kulturellen Unterschiede, der historischen und regionalen Eigenheiten, der geistigen und physischen Schöpferkraft der Menschheit, kurzum: eine Landkarte der Vielfalt der Kulturen und der Schönheit und Einzigartigkeit dieses Planeten. Alle diese Stätten zeigen uns die Schwäche und Zerbrechlichkeit, die Schönheit und den Glanz unserer Welt vor der dunklen Unendlichkeit des Universums.

Als Objekte des Weltinteresses erfahren Welterbestätten große nationale und internationale Aufmerksamkeit in den Medien, und dies nicht nur anlässlich von besonders medial wirksamen Konflikten (Dresden, Köln etc.). Wenn ich von einer "Landkarte (der kulturellen Vielfalt)" spreche, dann meine ich das durchaus wörtlich - also auch im Sinne der geographischen und touristisch nutzbaren Landkarte. Welterbestätten sind in allen Reiseführern dieser Welt verzeichnet. Wie aber verhalten sich Tourismus und Welterbe-Status zueinander? Um es vorwegzunehmen: Tourismus und Welterbe-Status können sich im Idealfall gegenseitig ergänzen und einander nützen:

Das Prädikat "Welterbe" ist zweifellos als Marketinginstrument international wirksam. Studien zeigen, dass die UNESCO ein durchweg positives Image hat und mit kultureller Einzigartigkeit gleichgesetzt wird; keine andere Kulturmarke kann sich mit der internationalen Präsenz, Bekanntheit und Akzeptanz der UNESCO vergleichen. Das UNESCO-Label hat einen weltweit unbestrittenen Markenwert, der sich für den Tourismus auszahlt - ideell und finanziell. Der UNESCO-Titel bringt nicht nur zusätzliche Gäste aus dem Inland, sondern auch solche aus zum Teil sehr fernen Regionen der Erde.

Es steht außer Frage, dass der Welterbe-Titel einen positiven Einfluss auf die lokale Wirtschaft hat. Zwar lässt er sich nur schwer in Heller, Pfennig oder Cent berechnen und ist von Stätte zu Stätte unterschiedlich. Der Zugewinn an touristischem Prestige kann sich aber als positiver ökonomischer Anreiz auswirken, übrigens auch zur Verbesserung der Lebensqualität der Anwohner.

Niemand sollte jedoch die UNESCO-Auszeichnung ausschließlich als MarketingInstrument missverstehen: Ungelenkter und ausufernder Tourismus hat negative Auswirkungen auf die natürliche Umwelt und auf die von ihr lebenden Gemeinschaften. Venedig mit seinen weniger als 100.000 Einwohnern, die alljährlich von 18 Millionen Besuchern heimgesucht werden, ist dafür ein beredtes Beispiel. Tourismus kann zu einem erheblichen Belastungsfaktor werden, der auch den Welterbe-Status gefährden kann. Denn bei aller Freude über den Erfolg der Welterbe-Konvention sind es doch oft 
die ganz besondere Atmosphäre, die Aura und der Zauber der Welterbestätten, die durch zu viel Tourismus als erstes gefährdet sind. Auf der Chinesischen Mauer, in Machu Picchu, in Angkor Wat, aber auch an manchen Tagen im Kölner Dom kann man erleben, was ich damit meine. So hat auch das beste Programm zum Schutz unseres Kultur- und Naturerbes seinen unvermeidlichen Preis.

Daher sollte die Tourismusbranche zusammen mit den vor Ort Verantwortlichen wirksame Mechanismen und Verhaltenskodizes entwickeln, um Welterbestätten vor unkontrollierter touristischer Nutzung zu bewahren. Es geht um Partnerschaftsmodelle, die über den kurzfristigen Gewinn hinaus den langfristigen Schutz einer Stätte gewährleisten und irreparable Schäden von vorneherein vermeiden helfen. Ich wiederhole: Es geht über den kurzfristigen Gewinn hinaus zuerst und zuvörderst immer um den langfristigen Schutz einer Stätte.

Außerdem sollte Welterbe-Tourismus den Ansprüchen nachhaltiger Entwicklung, wie von der Welttourismusorganisation (WTO) definiert, gerecht werden: Er sollte also im Umgang mit den Ressourcen ökologisch verantwortlich sein und der Völkerverständigung dienen. Alle Maßnahmen sollten unter Mitwirkung der einheimischen Bevölkerung geschehen. Der Respekt vor den heute dort lebenden Menschen sollte genau so groß sein wie die Bewunderung für künstlerische Leistungen der Vergangenheit oder die Freude an der eigenen Neugier. Wenn also der ökonomische Nutzen des WelterbeStatus für die Tourismusbranche erheblich ist, dann ist es umgekehrt auch ein legitimes Anliegen, vor Ort einen Teil der durch den Tourismus erwirtschafteten Mittel in den Schutz der Welterbestätten zu investieren.

Neben den finanziellen und ökonomischen Aspekten gibt es aber auch andere Ansprüche, die an den Welterbe-Tourismus geknüpft werden müssen: Der Tourismus an Welterbestätten muss Nachhaltigkeit in ihrer sozialen, ökonomischen und ökologischen Dimension berücksichtigen. Dies bedeutet etwa Rücksichtnahme auf die lokale Bevölkerung, ein umfassendes Angebot an Regionalprodukten in Gastronomie und Hotellerie wie auch der nachhaltige Umgang mit begrenzten Ressourcen. Es gilt, die Bedürfnisse der Gäste und der lokalen Bevölkerung mit denen des Natur- und Umweltschutzes zu verbinden und dabei eine langfristig wirtschaftliche sowie sozial verträgliche Entwicklung anzustreben. Auf diese Weise trägt nachhaltiger Tourismus erheblich zu einer dauerhaften Wertschöpfung und zum Wohlstand der Bevölkerung bei.

Konkret bedeutet nachhaltiger Tourismus, dass er die heutigen Bedürfnisse der Touristen und Gastregionen befriedigt, während er die Zukunftschancen wahrt und erhöht. Die Bedürfnisse der Touristen, künstlerische Leistungen der Vergangenheit am Welterbe zu erleben, und die der lokalen Bevölkerung, in einer Welterbe-Altstadt zum Beispiel ihren Alltag zu gestalten, müssen in keinem Widerspruch stehen. Voraussetzung dafür ist, dass die lokale Bevölkerung eingebunden wird in die Gestaltung des Tourismus.

Zu den häufigsten Umweltbelastungen gehört der Straßen- und Luftverkehr. Neben einer erheblichen Lärmbelästigung für die Bürger hinterlässt er auch seine Spuren an 
Kultur- und Naturerbestätten - man denke nur an die verrußten Häuserfronten in Großstädten. Fahrradrouten statt Hop-on-hop-off-Busse sind ein guter Anfang, ebenso ein umfassendes Angebot für umweltschonende Anreisewege.

Die Stätten ihrerseits sollten Weltoffenheit signalisieren und mit Partnern in anderen Kontinenten kooperieren. Denn Tourismus ist auch eine Möglichkeit, das Weltkulturund Weltnaturerbe in seiner Authentizität für viele Menschen erfahrbar zu machen und sie für die Werte des Welterbes und der UNESCO zu sensibilisieren. Der klassische Zielkonflikt zwischen touristischer Nutzung und Naturschutz oder Denkmalpflege tritt bei den Welterbestätten oft in verschärfter Form auf. Der Konflikt mag notorisch sein, unvereinbar oder unüberbrückbar sind die Interessenslagen nicht. Tourismus ist vielmehr eine Möglichkeit, das Kultur- und Naturerbe der Welt für viele Menschen sichtbar und erlebbar zu machen. Die Verwirklichung eines nachhaltigen Tourismus ist ein kontinuierlicher Prozess. Er sollte ein hohes Maß an Zufriedenheit und sinnvolle Erlebnisse für Touristen gewährleisten und sie gleichzeitig für Fragen der Nachhaltigkeit sensibilisieren.

In diesem Sinne arbeitet die UNESCO derzeit an einem neuen internationalen Instrument, dem "World Heritage and Sustainable Tourism Program”. Bislang wurde noch nichts von der Generalkonferenz beschlossen, aber es ist sicher, dass in wenigen Jahren die "guidelines", die Richtlinien für das Welterbe, ergänzt werden um die neuen Aspekte des nachhaltigen Tourismus. In einem der Entwurfsdokumente heißt es, dass der Tourismus

if unplanned or not properly managed, [...] can be socially, culturally and economically disruptive, and have a devastating effect on fragile environments and local communities. [...] Inadequately managed tourism can have severe consequences compromising the OUV of the properties, depriving the local communities of benefits and potentially degrading the destination itself.

In diesem Entwurfsdokument für das "new and inclusive World Heritage and Sustainable Tourism Program" heißt es aber auch: "If undertaken responsibly, tourism can be a driver for preservation and conservation of cultural and natural heritage and a vehicle for sustainable development."

\section{Säule: Welterbe-Bildung}

Die Befassung mit dem Welterbe-Programm der UNESCO kann sich nicht mehr beschränken auf den konservatorischen denkmal- oder naturschützenden Aspekt der Welterbe-Konvention, geschweige denn auf den Tourismus. Als Teil des berühmten "Dialogs zwischen den Kulturen“ sind Welterbestätten auch Orte des interkulturellen Lernens und der interkulturellen Begegnung und Partnerschaft. Angesichts der Konflikte in dieser Welt wird diese dritte Säule des Welterbe-Programms der UNESCO, die Welterbe-Bildung, immer wichtiger. Welterbestätten sind Lernorte für ein zivilisiertes Miteinander. In ihnen wird beziehungsweise soll der Gedanke internationaler Partnerschaft sichtbar und erlebbar werden. Artikel 27 der Welterbe-Konvention ruft die Vertragsstaaten dazu auf, „unter Einsatz aller geeigneten Mittel, besonders durch Bildungs- 
und Informationsprogramme, die Würdigung und Achtung des in den Artikeln 1 und 2 bezeichneten Kultur- und Naturerbes durch ihre Völker zu stärken."

Demnach sind wir alle aufgefordert, die Welterbestätten für alle Menschen zugänglich zu machen und das Wissen darüber weiterzugeben. Der "außergewöhnlich universelle Wert", der Welterbestätten auszeichnet, ist oft nicht auf den ersten Blick für alle Menschen ersichtlich.

Friedrich Nietzsche hat zwischen drei Formen der Geschichtsbetrachtung unterschieden:

(1) einer antiquarischen, in der er nur "eine blinde Sammelwut, ein rastloses Zusammenscharren alles einmal Dagewesenen“ sieht. Diese Form der Geschichtsbetrachtung können wir hier außer Acht lassen.

(2) einer monumentalischen, für die die "großen Momente, die Sternstunden“ der Geschichte stehen. Beispiele dafür aus der Liste des Welterbes sind die Chinesische Mauer, die Pyramiden von Gizeh, das Tadsch Mahal, Machu Picchu, die Naturerbestätten Grand Canyon, das Great Barrier Reef und andere.

(3) einer kritischen Geschichtsbetrachtung, die die Vergangenheit "Vor Gericht zieht, sie peinlich inquiriert und endlich verurteilt" (zit. nach Harald Weinrich, "Lethe").

Stark vereinfachend können wir die Geschichte der Welterbe-Konvention als auf dem Weg von der zweiten Kategorie, der „monumentalischen“ zur dritten, der kritischen, zukunftsfähigen betrachten. Die jüngeren deutschen Welterbestätten - wie die Siedlungen der Berliner Moderne, die Zeche Zollverein und andere, sicherlich auch Hiroshima und Ausschwitz - gehören zweifelsfrei in diese dritte Kategorie.

Was können wir hier vor Gericht ziehen, inquirieren, verurteilen? Historische städtische Landschaften, wie sie die UNESCO sieht, sind zum Beispiel keine Ansammlung bedeutsamer Monumente, für die allein der Denkmalschutz zuständig wäre. Nein, sie sind lebende Organismen und lebendige Lebensräume für ihre Bewohner. In diesem Sinne sind Welterbestätten - wie zum Beispiel, aber nicht nur die historischen Städte und Landschaften - mit ihrer Schönheit, mit ihrem Geschichts- und Identifikationspotenzial, aber auch mit ihrer menschenfreundlichen Textur in Zukunft auch Lernorte für ein zivilisiertes Miteinander, für eine nachhaltige, das heißt generationenübergreifende Verantwortung unseren Mitmenschen und unserem Planeten gegenüber.

Aber kann es auch ein Geschichtsbewusstsein geben für Stätten wie zum Beispiel die Pfahlbauten, die von Zeiten künden, in denen es schriftliche Zeugnisse noch gar nicht gab? Einfacher gefragt: Was also können und sollten wir lernen an dieser "unsichtbaren“ Welterbestätte Pfahlbauten? Dies ist das Kernthema unserer Veranstaltung: Die Vermittlung eines "unsichtbaren“ Welterbes. Bei dieser Vermittlungsarbeit spielen im Rahmen einer Museums- und Erlebnispädagogik Ausstellungen und Führungen, Videos und Interviews eine zentrale Rolle, über die Emotionen und Eindrücke transportiert werden. 
Welterbe-Bildung sollte in ihrer Konzeption jedoch auch den Kerngedanken des Welterbes beinhalten: die interkulturelle Begegnung. Welterbestätten als Lernorte interkultureller Begegnung spiegeln verschiedenste internationale Einflüsse wider. Im Erleben des Welterbes werden die Grenzen aufgehoben. Als Teilhaber am Welterbe üben wir Education for global citizenship ein, Bildung zum Weltbürgertum.

Das Programm dieser Veranstaltung bietet zahlreiche Anknüpfungspunkte für das bisher Gesagte. Hinzuweisen ist etwa auf den Vortrag über "Prähistorische Pfahlbauten Unsichtbares Welterbe" (Helmut Schlichtherle) oder einen weiteren über "Sichtbarmachung und Vermittlung des Welterbes" (Klaus-Dieter Schnell), beide der dritten Säule zuzurechnen. „Den Welterbe-Tourismus in die Pflicht nehmen“ lautete der ursprüngliche Titel des Festvortrags von Kurt Luger (zweite Säule). Der Gedanke dahinter erinnert an einen Slogan, den ich am Wattenmeer oft gehört habe: "Das Watt ist event genug". Es geht also um die Inwertsetzung des "Outstanding Universal Value" statt um die schlichte ökonomische Verwertung des Welterbetitels. Auch Fragen der "seriellen Welterbestätten“ und der Zusammenarbeit über politische Grenzen hinweg werden auf der Tagung behandelt und Initiativen, die auf ein globales Netzwerk in der Welterbe-Bildung abzielen.

Die letzte Generalkonferenz der UNESCO hat sich in einer Resolution zur WelterbeBildung geäußert:

\footnotetext{
Culture can be misused as a political divider and a pretext to fuel violence among communities and nations. Such risk of politicization call for individual as well as collective vigilance. World heritage cultural sites should be used to teach both about the universality of human creativity and the diversity of cultures, in line with the principles formulated in the 2001 UNESCO Universal Declaration on Cultural Diversity [...] UNESCO should also make an effort to promote an open and tolerant understanding of world history, bearing in mind the relevance of its pedagogical use. If culture has the power to reinforce identities, it can also be a means to foster cooperation among nations, communities, and civilizations. UNESCO should spare no efforts in opening these doors between nations and in helping countries and communities to break free from stereotypical challenges.
}

Welterbe-Bildung fördert das Bewusstsein für Identität, den gegenseitigen Respekt, den Dialog, die Gemeinsamkeit in der Vielfalt, das Gefühl der Solidarität und den positiven Austausch zwischen den Kulturen.

Lassen Sie mich noch etwas hinzufügen: Das Welterbe-Programm zieht Eitelkeiten und Begehrlichkeiten an wie das Licht die Motten. Wir sollten daher auch etwas lernen, das im Deutschen mit einer etwas altmodischen Vokabel bezeichnet wird: Demut Demut vor den Meisterwerken der menschlichen Schöpferkraft, Demut vor den baulichen Zeugen der Geschichte und vor der Verletzlichkeit unseres Planeten. Zu wünschen wäre Demut aber auch angesichts der Fehlbarkeit menschlichen Urteils - denken Sie an den eingangs zitierten "Protest der Künstler“ gegen den Eiffelturm in Paris.

Ich wünsche Ihnen und uns allen eine interessante und spannende Tagung.

Dieter Offenhäußer

Stv. Generalsekretär und Pressesprecher der Deutschen UNESCO-Kommission 